\title{
To appear in
}

Physical Activity and Sport during the First Ten Years of Life: Multidisciplinary Perspectives,

Routledge

Physical Exercise and Cognitive Development:

Influence of Training Modalities and Characteristics

\section{David Moreau}

School of Psychology and Centre for Brain Research

The University of Auckland

New Zealand

Correspondence:

The University of Auckland

Science Centre

School of Psychology

Brain Dynamics Lab

23 Symonds Street

Auckland 1010, New Zealand

+6493737599 ext 83401

d.moreau@auckland.ac.nz 


\begin{abstract}
Physical exercise has been shown to relate to cognitive performance in a diverse range of populations, including children. Evidence has also indicated that the link is at least partly causal, with exercise interventions bringing a myriad of benefits to cognitive development. However, not all types of exercise regimens appear to be created equal—recent findings suggest that although ubiquitous, aerobic exercise might benefit from added components, either in terms of training modalities or cognitive demands. Here, I briefly review evidence for the general impact of physical exercise on cognitive development, before focusing in more detail on recent trends in exercise-induced cognitive enhancement. In particular, I emphasize the importance of exercise intensity, and argue that, given time constraints, aerobic exercise might not always be an optimal way to target the brain. I then discuss the potential for including additional components within exercise regimens, to enable more general, far-ranging, improvements. Finally, I conclude with a number of recommendations to facilitate the translation of research findings into practical situations.
\end{abstract}




\section{Introduction}

In the first decade of life, children are confronted with a number of challenging situations, often in environments they have never encountered before. This wide range of experiences shapes the teenagers they will grow into, in turn influencing the adults they will become. Exercise and sports occupy a central place among these experiences, helping children develop their motor skills, challenging cognitive processes in a unique and sustained way, and ensuring healthy habits are incorporated at a young age (see for example Singh et al., 2019).

Given its influence on brain and behaviour, can exercise be leveraged to maximize cognitive development? Recent trends of research across psychology and education have explored how malleable cognition is, and the extent to which individual cognitive abilities can be influenced by short interventions (see for a review Moreau, Macnamara, \& Hambrick, 2018). These include a wide variety of interventions, ranging from computerized training (Jaeggi, Karbach, \& Strobach, 2017), to videogame training (Green \& Bavelier, 2015), mindfulness meditation (Zeidan, Johnson, Diamond, David, \& Goolkasian, 2010), or even interacting with nature (Berman, Jonides, \& Kaplan, 2008), all of which have been purported to directly influence cognitive function. Yet the best way to exercise the brain arguably remains physical exercise. In the specific context of exercise interventions, researchers have investigated how short-term regimens (Chang, Labban, Gapin, \& Etnier, 2012; Moreau \& Chou, 2019) and long-term interventions (Northey, Cherbuin, Pumpa, Smee, \& Rattray, 2018) affect cognitive abilities. Although some work has started to apply this knowledge to developmental populations (see for a review Tomporowski \& Pesce, 2019), the power of exercise remains largely untapped in young populations.

In this chapter, I provide a brief review of the literature linking physical exercise and cognition, with a specific focus on cognitive development. I further discuss components of exercise that appear to influence this relation, such as exercise modalities (e.g., intensity, duration, frequency) and activity properties (e.g., novelty, diversity, complexity). Finally, I close with a discussion of practical recommendations, to help practitioners translate scientific findings in a way that can directly impact society.

\section{Physical Exercise and Cognition}

Physical exercise has long known to be associated with a myriad of benefits, including protective effects against a range of conditions including stroke, cancer, and 
cardiovascular diseases (Blair et al., 1995). By the same token, lack of exercise is linked to higher risks for disorders such as autism, schizophrenia, and attention deficit/hyperactivity disorder (ADHD) (Penedo \& Dahn, 2005). Yet the impact of physical exercise goes beyond protective effects - it is also associated with better cognitive performance on tasks of working memory (Moreau, 2013), cognitive control (Moreau, Kirk, \& Waldie, 2017), executive function (Moreau \& Chou, 2019), and spatial ability (Moreau, 2012; Moreau, Mansy-Dannay, \& Clerc, 2011). Importantly, the link is (at least partly) causal, a point that has been demonstrated with interventions showing enhanced cognitive performance post-exercise in academic and professional settings (Castelli, Hillman, Buck, \& Erwin, 2007; Coe, Pivarnik, Womack, Reeves, \& Malina, 2006; Keeley \& Fox, 2009; Moreau et al., 2017), better regulation of emotions (Blumenthal et al., 1991), and higher quality of life (Cancela Carral \& Ayán Pérez, 2007).

At the functional level, work by Chaddock-Heyman and colleagues (2013) has reported decreases in neural activity in the right anterior prefrontal cortex while children were engaged in cognitive control tasks after a one-year exercise intervention. These changes in neural activity were accompanied with cognitive improvements measured at the behavioral level. Note, however, that not all studies find decreases in neural activity associated with exercise interventions; for example, Davis and colleagues (2011) reported increases in prefrontal activity following a 13-week intervention program. These mixed findings suggest that complex factors may be associated with the neural substrates of exercise-induced cognitive improvements, in line with more general findings in the field of neuroscience (e.g., $\mathrm{Xu}$, Calhoun, \& Potenza, 2015). Irrespective of the specific neural effects of exercise, however, behavioral improvements post-interventions remain typical (but see Young, Angevaren, Rusted, \& Tabet, 2015).

At the structural level, physical exercise generally promotes neurogenesis (van Praag, Kempermann, \& Gage, 1999; Vivar, Potter, \& van Praag, 2013), and enhances a number of other processes such as neuronal survival (Vaynman, Ying, Yin, \& Gomez-Pinilla, 2006) and brain vascularization (Colcombe et al., 2006). Structural studies using Diffusion Tensor Imaging (DTI) have shown that physical exercise interventions in populations of children lead to greater white-matter integrity, especially in the uncinate fasciculus (Schaeffer et al., 2014) and the superior longitudinal fasciculus (Krafft et al., 2014), two fundamental networks connecting the limbic system and the prefrontal cortex, and the temporal and parietal lobes, 
respectively. These findings are corroborated by observational evidence, for example showing white-matter integrity differences between children of various fitness levels (Chaddock-Heyman et al., 2014). Exercise also upregulates neurotransmitter concentrations, as well as key mediator of the relation between exercise and cognition such a brain-derived neurotrophic factor (BDNF), which concentration increases in specific parts of the brain such as the hippocampus post-exercise (Neeper, Gómez-Pinilla, Choi, \& Cotman, 1996). This post-exercise increase appears within minutes and can last several weeks (Berchtold, Patrick Kesslak, Pike, Adlard, \& Cotman, 2001). Interestingly, higher BDNF concentrations are especially pronounced in specific regions of the brain such as the hippocampus, the caudal neocortex and the dentate gyrus - areas that are not primarily involved in motor control but in general cognitive function (Neeper et al., 1996). This finding supports the notion that physical exercise has a broad impact on the brain, above and beyond sensorimotor areas, and including brain regions involved in complex cognition.

Overall, the aforementioned literature reports potent effects of exercise on the brain. Yet, this line of work almost exclusively focuses on aerobic exercise, a specific type of moderate-intensity, sustained regimens that is popular but possibly not representative of typical exercise, especially in younger populations. Historically, this has followed on from animal research that focused on forms of exercise resembling human aerobic effort (e.g., Fuchs \& Gould, 2000; van Praag et al., 1999). However, recent work has shown that alternatives have great potential. In the following section, we describe work that has used regimens that may be more ecological, and thus might better translate to practical implementations.

\section{Critical Components of Exercise}

Beyond the general trend linking physical exercise to brain function and cognition, other research has further explored how varying components of exercise affects its impact on brain and mind. These components can be divided into two broad categories: the training modalities of exercise, such as intensity, duration and frequency, and the characteristics of the activity relative to each individual, for example, its novelty, diversity, or complexity.

Although early work on the link between brain and exercise has focused on aerobic exercise, novel trends have since emerged. For example, recent work in our group has shown that interventions involving high-intensity training — short bursts of exercise, repeated a number of times and interleaved with rest — could allow substantial improvements, 
comparable to those of longer, less intense regimens (Moreau \& Chou, 2019; Moreau et al., 2017). In a six-week randomized controlled intervention, we found that high-intensity training elicited improvements in cognitive control and working memory constructs assessed via a multitude of tasks (Moreau et al., 2017). These findings are consistent with literature showing the clear physiological effect of high-intensity training regimens (Rognmo, Hetland, Helgerud, Hoff, \& Slørdahl, 2004) and the health benefits associated with this type of regimen (Costigan, Eather, Plotnikoff, Taaffe, \& Lubans, 2015). Further work in our group demonstrated that the acute effects elicited by exercise are comparable across intensities (Moreau \& Chou, 2019), thus emphasizing the role high-intensity exercise might play to maximize impact when time constraints are important—-for example, in the context of academic curriculums.

Related work has explored the influence of duration and frequency on exercise-induced benefits, though findings in this area remain equivocal. For example, a resistance-training intervention study compared two frequencies, once and twice a week on cognitive function, and found comparable cognitive improvements for both groups (Liu-Ambrose et al., 2010) — that is, the difference in frequencies did not translate into differences in cognitive outcomes. Similarly, a meta-analysis on the relationship between exercise and cognitive function in older adults found no evidence of a dose-dependent relationship (Sanders, Hortobágyi, la Bastide-van Gemert, van der Zee, \& van Heuvelen, 2019). It is important to note that further research is needed, given the inconclusive nature of these findings, and that those specific results may not necessarily apply to younger populations. Dose-response studies with exercise are notoriously difficult to conduct, because they rely on very large sample sizes to maintain adequate statistical power (e.g., Moreau, Kirk, \& Waldie, 2016).

In addition to exercise modalities, researchers have investigated the influence of the specific characteristics of physical activities (Tomporowski, McCullick, Pendleton, \& Pesce, 2015). Within this body of work, findings suggest that novelty, diversity and complexity are important components to factor into exercise regimens - these components can be actively manipulated by leveraging the rich nature of physical activities, which offer a range of situations, adapted to all levels of expertise. Content can thus be adapted to the specific level of expertise of each child, therefore keeping them engaged (with content that is not too difficult) yet interested (with content that is challenging enough). Movements can range from 
simple, automated coordination, to complex, challenging skills that require sustained attention. This natural progression provides a continuum of activities for which novelty and difficulty can be manipulated, based on individual strengths and limitations.

The idea that complexity is key to learning and brain development is supported by animal models (Anderson, Sisti, Curlik, \& Shors, 2011; Curlik \& Shors, 2013), showing for instance that combining mental and physical training helps ensure neuronal recruitment and overall mental health, to a greater extent that either mental training or physical exercise in isolation. These results are consistent with studies in humans, including research in our group. In 2015, we showed that activities that combine cognitive challenges with exercise provide a superior mental workout that either cognitive or exercise training alone (Moreau, Morrison, \& Conway, 2015), suggesting that a key component of successful training program targeting brain and mind is the combination of exercise with cognitive challenges (Moreau, 2015; Moreau \& Conway, 2013, 2014). Importantly, this is also in line with other research in the field, including work in younger populations; for example, Pesce and colleagues (Pesce, 2012; Pesce \& Ben-Soussan, 2016; Pesce et al., 2016) found that combining complex motor training with exercise in physical education classes can have additive benefits on executive function. Similarly, a large clinical trial by the same group found evidence for the influence of motor skill acquisition on inhibitory control, adding to the growing evidence that the effect of physical exercise on cognitive function is best complemented with cognitive challenges (see for a review Tomporowski \& Pesce, 2019).

Specific comparisons of complex motor training with the isolated components they include is also in line with research showing that sports training such as wrestling or martial arts has a greater impact on cognition that simpler forms of exercise. In a randomized experiment, our group found that exercise interventions were more beneficial when they were based on wrestling - a complex motor activity — than when based on running, a simpler form of exercise (Moreau, Clerc, Mansy-Dannay, \& Guerrien, 2012). Similarly, Lakes and colleagues (2013) found that a 9-month martial arts program had a greater impact on executive function in children than a traditional physical education class component. Assessments of executive function included working memory capacity, cognitive flexibility and inhibitory control, major processes in most everyday activities, and associated with achievement in academic domains (Diamond \& Lee, 2011). As Diamond and Lee (2011) wrote: "To improve executive functions, focusing narrowly on them may not be as effective 
as also addressing emotional and social development (as do curricula that improve executive functions) and physical development (shown by positive effects of aerobics, martial arts, and yoga)" (abstract). These aspects are possibly best addressed via ecological activities (Moreau \& Conway, 2014)

Ecological environments such as those proposed by physical activities can also help blend physical and cognitive demands by introducing a shortage of resources in a number of domains, especially physiological and cognitive. With higher intensities of exercise, neural systems have to function with fewer resources, such as oxygen and nutrients, forcing them to be more efficient (see for a review Moreau \& Conway, 2013). This aspect makes physical activities particularly challenging - both the physical and cognitive demands, often offered separately or sequentially in training experiments, are in this case concurrent, providing the basis for demanding regimens. Importantly, optimal environments can differ across individuals — not everyone will benefit equally from a given regimen (if at all). In this context, proposing the right content for a given child requires precise knowledge of cognitive development, cognitive improvement, responses to exercise and other characteristics (e.g., neural, physiological, genetic) at the individual level. This is the first step toward personalized exercise interventions, a goal that should be balanced with the downsides of individual content for group dynamics and social interactions. In any case, further research will allow more precise predictions about what can be achieved via exercise, and how it can be harnessed to enrich cognitive development in childhood.

\section{Practical Recommendations}

Translating research findings into practical advice can prove challenging, not least because findings in this body of research remain mixed. There are, however, a number of general findings that are arguably consensual. Below, I make five simple recommendations for those who wish to harness the power of physical exercise for the health of their children, students, or trainees.

1) Encourage exercise. First, it is important to recognize that physical exercise, no matter the modality, is a fundamental improvement over sedentary behaviors (e.g., Ahamed et al., 2007). Exercise is associated with an extraordinary number of benefits, in a wide variety of domains such as cognitive, psychological, and social. This means that the additional recommendations that follow-perhaps at the exception of the final one-are inherently less important than the act of exercising itself, which should 
remain the primary goal. Provided one has the willingness and ability to exercise, a number of other recommendations are helpful in my view.

2) Propose novel activities. When seeking cognitive improvements via exercise, it is important to remember that components that we know are beneficial to the health of our brains and minds, such as novelty and diversity, are key to include in exercise regimens. These components may be unusual or seem counterintuitive in sports, because they seemingly run counter to what is needed to become an expert-focusing on a given skill or set of skills and their repetition to achieve mastery (Ericsson, Krampe, \& Tesch-Römer, 1993; Macnamara, Moreau, \& Hambrick, 2016). However, the same components do make sense in a brain training framework, where improvements are elicited when content is challenging. The good news is, whereas adults often find it difficult to wander outside their comfort zone when exercising, children are used to facing new challenges in unusual settings. Thus, proposing novel challenges in unusual environments is often more straightforward with children.

3) Teach children to embrace difficulty. Many of the new generation of activities children are naturally drawn to involve immediate gratification. Smart technologies around them propose endless supplies of rewards, which can become very addictive. In this context, it is often difficult for teachers or educators to motivate students to engage with activities or skills that take some time to master or even just to feel comfortable with. Yet, the same children who might take a little more time to get on board do eventually find exercise appealing and rewarding, especially if exposure is gradual.

Tips include keeping exercise fun and engaging, for example with play (Tomporowski \& Pesce, 2019), proposing activities that are challenging but not overly difficult (Moreau, 2015), and using shorter forms of exercise when suitable (Moreau et al., 2017).

4) Vary intensity. It can be helpful to explore different ranges of exercise intensities. Long workouts are fine, but do make sure intensity is a feature in a number of those workouts. Research suggests that shorter workouts can elicit similar effects to longer forms of exercise, while being less time-consuming (Moreau et al., 2017). Similarly, proposing a range of physical activities that tap different physiological and cognitive domains is the best way to ensure children are stimulated, constantly challenged, and remain interested. 
5) Promote enjoyment. An important component of exercise and sports is that they are enjoyable to most children. Indeed, some research suggests that this component might be critical to ensure exercise has a positive effect on brains and minds (Yuede et al., 2009). Enjoyment is often favored by playful activities, yet these do not necessarily need to be structured: young children mostly enjoy solitary play, parallel play, or cooperative play that is not constrained by exterior rules (Bjorklund \& Brown, 1998). As children grow older, and with improvements in motor coordination and skills, they can be introduced to sports, in which exercise is more constrained and codified. Irrespective of the form of exercise favored, enjoyment is critical because it indicates a fundamental agreement between behavior that is healthy and what a child wants, thus allowing parents, teachers and educators to propose activities that are in the best interest of younger generations.

\section{Concluding Remarks}

In this chapter, I have briefly reviewed the impact of physical exercise on cognition and cognitive development, before exploring components such as exercise modalities and activity characteristics. Recent trends of research have shown that exercise need not be long and cumbersome - important benefits can be observed with shorter, and possibly more enjoyable, regimens. Harnessing the different aspects exercise has to offer provides parents, teachers, and educators with the tools to fulfill each individual's full potential. Exercise habits formed early on can help raise generations of children who not only benefit from good practices as they grow, but also carry these healthy habits well into adulthood.

Our understanding of how and when exercise can help cognitive development is still in its infancy. Among the components that remain to be explored are the dynamics of brain and behavior in the context of exercise-induced cognitive improvements - for example, how long-lasting improvements are, how these interact with child development, and the extent to which acute improvements are different from long-term enhancement. Answering these questions will require fine-grained methods and large samples, possibly via large-scale preregistered collaborations. This endeavor is worth pursuing, however, as it will facilitate translation to various populations and, eventually, personalized programs based on individual traits and characteristics. 
TRAINING MODALITIES AND CHARACTERISTICS

10

\section{Acknowledgements}

At the time of writing, the author was supported by a Marsden grant from the Royal Society of New Zealand, funding from the Neurological Foundation of New Zealand, the Ministry of Science and Technology (MST) in Taiwan, a University of Auckland Early Career Research Excellence Award (ECREA) and the Faculty Research and Development Fund (FRDF). 
TRAINING MODALITIES AND CHARACTERISTICS

11

\section{References}

Ahamed, Y., Macdonald, H., Reed, K., Naylor, P.-J., Liu-Ambrose, T., \& McKay, H. (2007).

School-based physical activity does not compromise children's academic performance.

Medicine and Science in Sports and Exercise, 39(2), 371-376.

Anderson, M. L., Sisti, H. M., Curlik, D. M., \& Shors, T. J. (2011). Associative learning increases adult neurogenesis during a critical period. European Journal of Neuroscience, Vol. 33, pp. 175-181. https://doi.org/10.1111/j.1460-9568.2010.07486.x

Berchtold, N. C., Patrick Kesslak, J., Pike, C. J., Adlard, P. A., \& Cotman, C. W. (2001). Estrogen and exercise interact to regulate brain-derived neurotrophic factor mRNA and protein expression in the hippocampus. European Journal of Neuroscience, Vol. 14, pp. 1992-2002. https://doi.org/10.1046/j.0953-816x.2001.01825.x

Berman, M. G., Jonides, J., \& Kaplan, S. (2008). The cognitive benefits of interacting with nature. Psychological Science, 19(12), 1207-1212.

Bjorklund, D. F., \& Brown, R. D. (1998). [Review of Physical play and cognitive development: integrating activity, cognition, and education]. Child development, 69(3), 604-606.

Blair, S. N., Kohl, H. W., 3rd, Barlow, C. E., Paffenbarger, R. S., Jr, Gibbons, L. W., \& Macera, C. A. (1995). Changes in physical fitness and all-cause mortality. A prospective study of healthy and unhealthy men. JAMA: The Journal of the American Medical Association, 273(14), 1093-1098.

Blumenthal, J. A., Matthews, K., Fredrikson, M., Rifai, N., Schniebolk, S., German, D., ... Rodin, J. (1991). Effects of exercise training on cardiovascular function and plasma lipid, lipoprotein, and apolipoprotein concentrations in premenopausal and postmenopausal women. Arteriosclerosis and Thrombosis: A Journal of Vascular 
TRAINING MODALITIES AND CHARACTERISTICS

12

Biology / American Heart Association, 11(4), 912-917.

Cancela Carral, J. M., \& Ayán Pérez, C. (2007). Effects of high-intensity combined training on women over 65. Gerontology, 53(6), 340-346.

Castelli, D. M., Hillman, C. H., Buck, S. M., \& Erwin, H. E. (2007). Physical Fitness and Academic Achievement in Third- and Fifth-Grade Students. Journal of Sport and Exercise Psychology, Vol. 29, pp. 239-252. https://doi.org/10.1123/jsep.29.2.239

Chaddock-Heyman, L., Erickson, K. I., Holtrop, J. L., Voss, M. W., Pontifex, M. B., Raine, L. B., ... Kramer, A. F. (2014). Aerobic fitness is associated with greater white matter integrity in children. Frontiers in Human Neuroscience, 8, 584.

Chang, Y. K., Labban, J. D., Gapin, J. I., \& Etnier, J. L. (2012). The effects of acute exercise on cognitive performance: a meta-analysis. Brain Research, 1453, 87-101.

Coe, D. P., Pivarnik, J. M., Womack, C. J., Reeves, M. J., \& Malina, R. M. (2006). Effect of physical education and activity levels on academic achievement in children. Medicine and Science in Sports and Exercise, 38(8), 1515-1519.

Colcombe, S. J., Erickson, K. I., Scalf, P. E., Kim, J. S., Prakash, R., McAuley, E., ... Kramer, A. F. (2006). Aerobic exercise training increases brain volume in aging humans. The Journals of Gerontology. Series A, Biological Sciences and Medical Sciences, 61(11), 1166-1170.

Costigan, S. A., Eather, N., Plotnikoff, R. C., Taaffe, D. R., \& Lubans, D. R. (2015).

High-intensity interval training for improving health-related fitness in adolescents: a systematic review and meta-analysis. British Journal of Sports Medicine, Vol. 49, pp. 1253-1261. https://doi.org/10.1136/bjsports-2014-094490

Curlik, D. M., \& Shors, T. J. (2013). Training your brain: Do mental and physical (MAP) training enhance cognition through the process of neurogenesis in the hippocampus? 
TRAINING MODALITIES AND CHARACTERISTICS

13

Neuropharmacology, Vol. 64, pp. 506-514.

https://doi.org/10.1016/j.neuropharm.2012.07.027

Davis, C. L., Tomporowski, P. D., McDowell, J. E., Austin, B. P., Miller, P. H., Yanasak, N.

E., ... Naglieri, J. A. (2011). Exercise improves executive function and achievement and alters brain activation in overweight children: a randomized, controlled trial. Health Psychology: Official Journal of the Division of Health Psychology, American Psychological Association, 30(1), 91-98.

Diamond, A., \& Lee, K. (2011). Interventions shown to aid executive function development in children 4 to 12 years old. Science, 333(6045), 959-964.

Ericsson, K. A., Krampe, R. T., \& Tesch-Römer, C. (1993). The role of deliberate practice in the acquisition of expert performance. Psychological Review, 100(3), 363-406.

Fuchs, E., \& Gould, E. (2000). In vivo neurogenesis in the adult brain: regulation and functional implications: Neurogenesis in adulthood. The European Journal of Neuroscience, 12(7), 2211-2214.

Green, C. S., \& Bavelier, D. (2015). Action video game training for cognitive enhancement. Current Opinion in Behavioral Sciences, 4, 103-108.

Jaeggi, S. M., Karbach, J., \& Strobach, T. (2017). Editorial Special Topic: Enhancing Brain and Cognition Through Cognitive Training. Journal of Cognitive Enhancement : Towards the Integration of Theory and Practice, 1(4), 353-357.

Keeley, T. J. H., \& Fox, K. R. (2009). The impact of physical activity and fitness on academic achievement and cognitive performance in children. International Review of Sport and Exercise Psychology, 2(2), 198-214.

Krafft, C. E., Schwarz, N. F., Chi, L., Weinberger, A. L., Schaeffer, D. J., Pierce, J. E., ... McDowell, J. E. (2014). An 8-month randomized controlled exercise trial alters brain 
TRAINING MODALITIES AND CHARACTERISTICS

14

activation during cognitive tasks in overweight children. Obesity, 22(1), 232-242.

Lakes, K. D., Bryars, T., Sirisinahal, S., Salim, N., Arastoo, S., Emmerson, N., ... Kang, C. J. (2013). The Healthy for Life Taekwondo Pilot Study: A Preliminary Evaluation of Effects on Executive Function and BMI, Feasibility, and Acceptability. Mental Health and Physical Activity, 6(3), 181-188.

Liu-Ambrose, T., Nagamatsu, L. S., Graf, P., Beattie, B. L., Ashe, M. C., \& Handy, T. C. (2010). Resistance training and executive functions: a 12-month randomized controlled trial. Archives of Internal Medicine, 170(2), 170-178.

Macnamara, B. N., Moreau, D., \& Hambrick, D. Z. (2016). The Relationship Between Deliberate Practice and Performance in Sports: A Meta-Analysis. Perspectives on Psychological Science: A Journal of the Association for Psychological Science, 11(3), $333-350$.

Moreau, D. (2012). The role of motor processes in three-dimensional mental rotation: Shaping cognitive processing via sensorimotor experience. Learning and Individual Differences, 22(3), 354-359.

Moreau, D. (2013). Motor expertise modulates movement processing in working memory. Acta Psychologica, 142(3), 356-361.

Moreau, D. (2015). Brains and Brawn: Complex Motor Activities to Maximize Cognitive Enhancement. Educational Psychology Review, 27(3), 475-482.

Moreau, D., \& Chou, E. (2019). The Acute Effect of High-Intensity Exercise on Executive Function: A Meta-Analysis. Perspectives on Psychological Science, p. 174569161985056. https://doi.org/10.1177/1745691619850568

Moreau, D., Clerc, J., Mansy-Dannay, A., \& Guerrien, A. (2012). Enhancing Spatial Ability Through Sport Practice. Journal of Individual Differences, 33(2), 83-88. 
TRAINING MODALITIES AND CHARACTERISTICS

15

Moreau, D., \& Conway, A. R. A. (2013). Cognitive enhancement: a comparative review of computerized and athletic training programs. International Review of Sport and Exercise Psychology, 6(1), 155-183.

Moreau, D., \& Conway, A. R. A. (2014). The case for an ecological approach to cognitive training. Trends in Cognitive Sciences, 18(7), 334-336.

Moreau, D., Kirk, I. J., \& Waldie, K. E. (2016). Seven Pervasive Statistical Flaws in Cognitive Training Interventions. Frontiers in Human Neuroscience, Vol. 10. https://doi.org/10.3389/fnhum.2016.00153

Moreau, D., Kirk, I. J., \& Waldie, K. E. (2017). High-intensity training enhances executive function in children in a randomized, placebo-controlled trial. eLife, 6 . https://doi.org/10.7554/eLife.25062

Moreau, D., Macnamara, B. N., \& Hambrick, D. Z. (2018). Overstating the Role of Environmental Factors in Success: A Cautionary Note. Current Directions in Psychological Science, 0963721418797300.

Moreau, D., Mansy-Dannay, A., \& Clerc, J. (2011). Spatial ability and motor performance: assessing mental rotation processes in elite and novice athletes. International Journal of. Retrieved from https://psycnet.apa.org/record/2012-03633-002

Moreau, D., Morrison, A. B., \& Conway, A. R. A. (2015). An ecological approach to cognitive enhancement: complex motor training. Acta Psychologica, 157, 44-55.

Neeper, S. A., Gómez-Pinilla, F., Choi, J., \& Cotman, C. W. (1996). Physical activity increases mRNA for brain-derived neurotrophic factor and nerve growth factor in rat brain. Brain Research, Vol. 726, pp. 49-56. https://doi.org/10.1016/0006-8993(96)00273-9

Northey, J. M., Cherbuin, N., Pumpa, K. L., Smee, D. J., \& Rattray, B. (2018). Exercise 
TRAINING MODALITIES AND CHARACTERISTICS

16

interventions for cognitive function in adults older than 50: a systematic review with meta-analysis. British Journal of Sports Medicine, 52(3), 154-160.

Penedo, F. J., \& Dahn, J. R. (2005). Exercise and well-being: a review of mental and physical health benefits associated with physical activity. Current Opinion in Psychiatry, 18(2), $189-193$.

Pesce, C. (2012). Shifting the focus from quantitative to qualitative exercise characteristics in exercise and cognition research. Journal of Sport \& Exercise Psychology, 34(6), $766-786$.

Pesce, C., \& Ben-Soussan, T. D. (2016). “Cogito ergo sum” or “ambulo ergo sum”? New perspectives in developmental exercise and cognition research. In T. McMorris (Ed.), Exercise-cognition interaction: Neuroscience perspectives , (pp (Vol. 496, pp. 251-282). San Diego, CA, US: Elsevier Academic Press, $\mathrm{x}$.

Pesce, C., Masci, C., Marchetti, R., Vazou, S., Sääkslahti, A., \& Tomporowski, P. D. (2016). Deliberate play jointly benefits motor and cognitive development: Direct and indirect effects of cognitive stimulation by movement. Frontiers in Psychology, 7, 349.

Rognmo, Ø., Hetland, E., Helgerud, J., Hoff, J., \& Slørdahl, S. A. (2004). High intensity aerobic interval exercise is superior to moderate intensity exercise for increasing aerobic capacity in patients with coronary artery disease. European Journal of Cardiovascular Prevention and Rehabilitation: Official Journal of the European Society of Cardiology, Working Groups on Epidemiology \& Prevention and Cardiac Rehabilitation and Exercise Physiology, 11(3), 216-222.

Sanders, L. M. J., Hortobágyi, T., la Bastide-van Gemert, S., van der Zee, E. A., \& van Heuvelen, M. J. G. (2019). Dose-response relationship between exercise and cognitive function in older adults with and without cognitive impairment: A systematic review and 
TRAINING MODALITIES AND CHARACTERISTICS

17

meta-analysis. PloS One, 14(1), e0210036.

Schaeffer, D. J., Krafft, C. E., Schwarz, N. F., Chi, L., Rodrigue, A. L., Pierce, J. E., ...

McDowell, J. E. (2014). An 8-month exercise intervention alters frontotemporal white matter integrity in overweight children. Psychophysiology, Vol. 51, pp. 728-733. https://doi.org/10.1111/psyp.12227

Singh, A. S., Saliasi, E., van den Berg, V., Uijtdewilligen, L., de Groot, R. H. M., Jolles, J., ... Chinapaw, M. J. M. (2019). Effects of physical activity interventions on cognitive and academic performance in children and adolescents: a novel combination of a systematic review and recommendations from an expert panel. British Journal of Sports Medicine, 53(10), 640-647.

Tomporowski, P. D., McCullick, B., Pendleton, D. M., \& Pesce, C. (2015). Exercise and children's cognition: The role of exercise characteristics and a place for metacognition. Journal of Sport and Health Science, 4(1), 47-55.

Tomporowski, P. D., \& Pesce, C. (2019). Exercise, sports, and performance arts benefit cognition via a common process. Psychological Bulletin, Vol. 145, pp. 929-951. https://doi.org/10.1037/bu10000200

van Praag, H., Kempermann, G., \& Gage, F. H. (1999). Running increases cell proliferation and neurogenesis in the adult mouse dentate gyrus. Nature Neuroscience, 2(3), 266-270.

Vaynman, S. S., Ying, Z., Yin, D., \& Gomez-Pinilla, F. (2006). Exercise differentially regulates synaptic proteins associated to the function of BDNF. Brain Research, $1070(1), 124-130$.

Vivar, C., Potter, M. C., \& van Praag, H. (2013). All about running: synaptic plasticity, growth factors and adult hippocampal neurogenesis. Current Topics in Behavioral Neurosciences, 15, 189-210. 
TRAINING MODALITIES AND CHARACTERISTICS

18

Xu, J., Calhoun, V. D., \& Potenza, M. N. (2015). The absence of task-related increases in BOLD signal does not equate to absence of task-related brain activation. Journal of Neuroscience Methods, Vol. 240, pp. 125-127.

https://doi.org/10.1016/j.jneumeth.2014.11.002

Young, J., Angevaren, M., Rusted, J., \& Tabet, N. (2015). Aerobic exercise to improve cognitive function in older people without known cognitive impairment. Cochrane Database of Systematic Reviews , (4), CD005381.

Yuede, C. M., Zimmerman, S. D., Dong, H., Kling, M. J., Bero, A. W., Holtzman, D. M., ... Csernansky, J. G. (2009). Effects of voluntary and forced exercise on plaque deposition, hippocampal volume, and behavior in the Tg2576 mouse model of Alzheimer's disease. Neurobiology of Disease, 35(3), 426-432.

Zeidan, F., Johnson, S. K., Diamond, B. J., David, Z., \& Goolkasian, P. (2010). Mindfulness meditation improves cognition: evidence of brief mental training. Consciousness and Cognition, 19(2), 597-605. 